\section{Human health risk from hexachlorocyclohexane and dichlorodiphenyl- trichloroethane pesticides, through consumption of vegetables: estimation of daily intake and hazard quotients}

\author{
Bhupander Kumar, 1 \\ Virendra Kumar Verma, ${ }^{1}$ \\ Ashis Kumar Naskar, ${ }^{2}$ \\ Paromita Chakraborty, ${ }^{3}$ Sanjay Kumar, ${ }^{1}$ \\ Debapriyo Mukherjee ${ }^{2}$ \\ ${ }^{1}$ Central Pollution Control Board, East \\ Arjun Nagar, Delhi; ${ }^{2}$ Central Pollution \\ Control Board, Southern Conclave, \\ Kolkata; ${ }^{3}$ SRM Research Institute, SRM \\ University, Kattankulathur, India
}

\section{Abstract}

People are encouraged to consume more fresh vegetables due to the high nutritional value and fibre contents in vegetables. At the same time, due to pesticidal residue in vegetables, vegetable consumption is considered as an important route of pesticides uptake by human beings. Pesticides, particularly organochlorines such as Aldrin, heptachlor and isomers of dichlorodiphe nyltrichloroethane (DDT) and hexachlorocyclohexane $(\mathrm{HCH})$ are a major concern because of their persistence, bioaccumulation, toxicity, and long-range environmental transport ability. Hence information on such toxic contaminants in vegetables is very essential, due to human health concern. In order to assess the potential health risks associated with vegetable consumption due to pesticidal residues of DDT and $\mathrm{HCH}$, sixty four samples of commonly available vegetables were collected from a local market in West Bengal, India and analysed for DDT and $\mathrm{HCH}$. In this study, estimated average daily intake of HCHs and DDTs through vegetables was ranged between $5.0 \times 10^{-8}-9.1 \times 10^{-6} \mathrm{mg} \mathrm{kg}^{-1}$ day ${ }^{-1}$ with an average value of $2.0 \times 10^{-6} \mathrm{mg} \mathrm{kg}^{-1} \mathrm{day}^{-1}$. Human health risk was assessed in terms of incremental lifetime cancer risk and health hazard index was between $1.7 \times 10^{-8}-8.8 \times 10^{-6}$ and $1.5 \times 10^{-7}$ $1.7 \times 10^{-2}$, respectively. Generated results were compared with maximum residual levels and reference values, were within acceptable limits and suggested no health risk to the human populations. Therefore, an extensive study on vegetables with the inclusion of other persistent organic pollutants and toxic metals should be carried out to know the overall scenario of contamination of vegetables.

\section{Introduction}

Dichlorodiphenyltrichloroethane (DDT) and hexachlorocyclohexane $(\mathrm{HCH})$ are persistent bioaccumulative contaminants that are found ubiquitously in environment and biological matrices, including tissues of fish, birds and mammals. ${ }^{1-2}$ These toxic organic pollutants compounds can travel longer distance by long range atmospheric transport thereby affecting regions far from their release sources ${ }^{3}$ and therefore listed by the Stockholm Convention as persistent organic pollutants. ${ }^{4}$ The use of DDT and HCH has been banned or severely restricted in several countries, but these are still being produced, exported, and used in some countries due to their low cost and versatile uses. ${ }^{5,6}$

India is the fourth largest pesticide producer in the world after the US, Japan and China; domestic production of pesticides is approximately 85,000 MT and about 60,000 MT used annually. The organochlorine pesticides and Malathion accounts for about $71 \%$ of that used. ${ }^{7,8}$ DDT and technical $\mathrm{HCH}$ were earlier extensively used until 1989 and 1997, respectively. After this time, Government of India has withdrawn the use of DDT and HCH in agriculture, but restricted for public health purpose only as per World Health Organization guidelines. ${ }^{9}$

These compounds are much of concern for human health because of their wide variety of toxicity. Human exposure to DDT and $\mathrm{HCH}$ has been associated with hormonal-disruption, arthritis, diabetes and development of cancer. ${ }^{10-14}$ Robison et al. ${ }^{15}$ reported that technical DDT and $o, p^{\prime}$-DDT, the most estrogenic component of technical DDT, can support the growth of estrogen-dependent breast tumors in rats. Humans are exposed to these toxic contaminants, mainly through the consumption of contaminated food and occupational environments. Therefore, health risk assessment due to pesticidal residues has been conducted worldwide. ${ }^{16-18}$

Significant level of residues of HCHs and DDTs has been found in the biotic ${ }^{19-22}$ and abiotic ${ }^{23-28}$ compartment in the past and also in the recent years in India. Although study on $\mathrm{HCH}$ and DDT residues in vegetables have been carried out in India ${ }^{29-38}$ but, quantitative health risk from $\mathrm{HCH}$ and DDT in vegetables from India was rarely investigated. ${ }^{39}$ Majority of people in India are vegetarian and everyday people are being encouraged to consume more vegetables. Vegetables are important components of the human diet due to their high nutritional value and fiber contents. The domestic consumption of vegetables in the urban and rural areas has increased in the recent years. Thus Information on toxic con-
Correspondence: Bhupander Kumar, Central Pollution Control Board, East Arjun Nagar Delhi110032 India.

E-mail: bhupander_kumar@yahoo.com

Key words: dichlorodiphenyltrichloroethane, hexachlorocyclohexane, pesticides, vegetables, average daily dose, health risk, hazard index.

Contributions: the authors contributed equally.

Conflict of interests: the authors declare no potential conflict of interests.

Acknowledgements: the authors are highly thankful to the Chairman and Member Secretary, Central Pollution Control Board for support and encouragement to conduct the study. Mr. BR Naidu, then Incharge Zonal Office, Central Pollution Control Board, Kolkata is also acknowledged for providing the analytical facilities.

Received for publication: 1 April 2013.

Revision received: 6 May 2013.

Accepted for publication: 14 May 2013.

This work is licensed under a Creative Commons Attribution NonCommercial 3.0 License (CC BYNC 3.0).

(C) Copyright B. Kumar et al., 2013

Licensee PAGEPress, Italy

Journal of Xenobiotics 2013; 3:e6

doi:10.4081/xeno.2013.e6

taminants in vegetables is very essential for protection of human health, because the main non-occupational route of exposure to toxicants is through dietary intake. ${ }^{40}$ This study was undertaken to assess the daily dietary intake of $\mathrm{HCH}$ and DDT pesticide through vegetables and their potential health risks in terms of incremental lifetime cancer risk (ILCR) and non-cancer health hazard index (HI) for human beings. For this purpose, primarily we estimated lifetime average daily dose (LADD) of HCH and DDT isomers for human adults through vegetable intake. The LADD is the amount of a chemical intake by a person per $\mathrm{kg}$ of body weight per day which may suspect the adverse health effects when absorbed into the body over a long period of time, either through inhalation, ingestion or dermal contact. ${ }^{41}$ Secondary, human health risk in terms of upper-bound excess probabilistic ILCR and non-carcinogenic health hazards was estimated. ${ }^{41}$ ILCR is the probability of cancer development in a lifetime after a uniform exposure to any carcinogenic chemical, while non-carcinogenic health hazards as $\mathrm{HI}$ is the measure of the magnitude of exposure potential or a quantifiable potential for developing non-carcinogenic health effects after averaged exposure period. ${ }^{42}$ 


\section{Materials and Methods}

\section{Solvents, chemicals and standards}

High performance liquid chromatography grade solvents (acetone, methanol, dichloromethane, and hexane) and analytical grade chemicals (sodium sulphate, silver nitrate, potassium hydroxide, activated charcoal and sulphuric acid) were purchased from Merck (Delhi, India). Silica gel 60 (0.063-0.100 $\mathrm{mm}$ ) was from Supelco (Sigma-Aldrich Co., St. Louis, M0, USA). Silica gel and granular anhydrous sodium sulphate was cleaned in Soxhlet extractor with methanol, dichloromethane and acetone for $8 \mathrm{~h}$ each, then stored in air tight desiccator until use. Pesticide standard mixture stock was prepared by diluting the commercial standard solutions [ $p, p^{\prime}$-DDT, $o, p^{\prime}$ DDT, $p, p$ '-dichlorodiphenyldichloroethylene (DDE), $p, p$ '-dichlorodiphenyldichloroethane (DDD), $\alpha-\mathrm{HCH}$ and $\gamma-\mathrm{HCH}$ ] purchased from Supelco (Sigma-Aldrich) and the working standard solution was prepared by diluting the standard stock solutions in hexane.

\section{Sample collection and extraction}

The selected vegetables were collected from local markets of West Bengal, India. Vegetables namely, carrot (Daucus carota L.), cauliflower (Brassica oleracea), eggplant (Solanum melongena L), radish (Raphanus sativus L.), spinach (Spinacia oleracea L.), and tomato (S. lycopersicum L.) collected in clean polyethylene bags, labelled and transported on ice to the laboratory.

In the laboratory, after washing with double distilled water and wiping with tissue paper, vegetables cut into small pieces with a grater and thoroughly mixed. For extraction of pesticides, samples were mixed with anhydrous sodium sulphate and extracted three times with acetone on a mechanical shaker. The acetone extract was filtered with vacuum suction and concentrated to near $20-25 \mathrm{~mL}$ using a rotatory vacuum evaporator (Buchi, Essen, Germany). The concentrated extracts were subjected to liquid-liquid partitioning three times with hexane in a separatory funnel. Hexane layer with residues was collected by passing the sample through sodium sulphate and concentrated to $\sim 5 \mathrm{~mL}$.

\section{Extract clean-up for removal of pigments and interferences}

Concentrated sample extracts were primarily cleaned to remove pigments. Pigments were removed by passing through a chromatographic glass column containing activated charcoal and anhydrous sodium sulphate. Secondary, multilayered silica gel column chromatography was performed for removal of interfering compounds and other aliphatic compounds. Briefly, a chromatographic glass column $(300 \times 30 \mathrm{~mm})$ was packed from bottom up with $2.5 \mathrm{~g}$ activated silica gel, $4.0 \mathrm{~g}$ silver nitrate silica gel, $2.5 \mathrm{~g}$ activated silica gel, 4.0 basic silica gel, $2.5 \mathrm{~g}$ activated silica gel, $12.0 \mathrm{~g}$ acid silica and $5.0 \mathrm{~g}$ anhydrous sodium sulphate. Preliminary, before loading of sample extract, column was rinsed with $50 \mathrm{~mL}$-hexane. Subsequently, after loading the sample extract, elution of analytes was carried out using $170 \mathrm{~mL}$ hexane, and eluted extract was concentrated under slow stream of purified laboratory grade nitrogen using Rotatory Vacuum evaporator and Turbo Vap (Caliper, Princeton, NJ, USA) to 2.0 $\mathrm{mL}$. The concentrated clean extract was transferred to a sample vial and $1 \mu \mathrm{L}$ was manually injected onto a gas chromatograph equipped with an electron capture detector for quantification of $\mathrm{HCH}$ and DDT isomers.

\section{Sample analysis and analytical quality control}

The identification and quantification of DDTs ( $p, p^{\prime}$-DDT, $o, p^{\prime}$-DDT, $p, p^{\prime}$-DDE and $p, p^{\prime}$ DDD), and HCHs ( $\alpha-\mathrm{HCH}$ and $\gamma-\mathrm{HCH})$ in clean sample extracts was carried out, using a gas chromatograph (3400cx, Varian Star, Milpitas, CA, USA) equipped with ${ }^{63} \mathrm{Ni}$ electron capture detector. A capillary column (RTX-5) (30 $\mathrm{m} \times$ $0.25 \mathrm{~mm}$ id) coated with a $0.5 \mu \mathrm{m}$ of stationary phase (5\% diphenyl $95 \%$ dimethylpolysiloxane), was used for the separation of the compounds. The column oven temperature was initially maintained at $170^{\circ} \mathrm{C}$ and programmed to $220^{\circ} \mathrm{C}$ (at $7^{\circ} \mathrm{C} \mathrm{min}^{-1}$ ); temperature was again increased to $250^{\circ} \mathrm{C}$ (at $5^{\circ} \mathrm{C}$ min $^{-1}$ ) and held for $7.0 \mathrm{~min}$. The temperature of injector and detector was maintained at 250 and $325^{\circ} \mathrm{C}$ respectively. A purified laboratory grade, nitrogen gas was used as carrier (at $1.0 \mathrm{~mL} \mathrm{~min}^{-1}$ ) during all the analysis.

Reference standard solutions were used for instrument calibration and other quality control studies. Concentrations of target compounds were determined with an external standard method, comparing peak area in samples with the standards using. Peak identification was conducted with the accurate retention time of each standard. Before analysis, five levels of calibration curves of standards were prepared with $r^{2}$ value of 0.999 . Each sample was analyzed in duplicates and average values obtained from duplicate analysis of each sample was used in calculations. For quality assurance/quality control analyses, procedural blanks was analyzed to check for loss and cross contamination (analyte concentrations were below the detection limits). Random duplicate sample analysis (standard deviation $<10 \%$ ), calibration verification (standard deviation $<10 \%$ ), and matrix spiked study was carried out to maintain quality control of analysis. Sample spiked recoveries with known working standard solutions were assumed to be satisfactory for each compound, and those were in the ranges of $70-110 \%$. Method detection limits were obtained by processing replicate of a sample spiked with a quantity sufficient to produce a detectable response $(s / n>3)$ and multiplying the standard deviation by the $t_{\text {students }}$ value for processed replicates. Statistically calculated value of $<$ MDL (explain abbreviation) used for all HCH and DDT isomers was $<0.01 \mathrm{ng} \mathrm{g}^{-1}$. Levels below $0.01 \mu \mathrm{g} \mathrm{kg}^{-1}$ (reporting limit) were not used in calculation and considered as zero. Analytical results were not corrected for blank (<MDL) and recoveries (within acceptable range), and reported on wet weight basis. Further, it may be worthy to mention that our laboratory had been participating in proficiency testing exercises conducted by international agencies with satisfactory score of performance for organochlorine pesticides including HCHs and DDTs.

\section{Human health risk assessment}

Considering the toxicological effects of HCHs and DDTs, it is important to investigate the potential exposure of human through dietary intakes. In this study, HCHs and DDTs intake through vegetables was estimated as LADD. ILCR and non-cancer health hazard [hazard quotient (HQ)] to humans was assessed using the recommended risk assessment guidance: 41

$\mathrm{LADD}\left(\mathrm{mg} \mathrm{kg}^{-1} \mathrm{day}^{-1}\right)=(\mathrm{Cs} \times \mathrm{IR} \times \mathrm{F} \times \mathrm{EF} \times \mathrm{ED}) /$

$(\mathrm{BW} \times \mathrm{AT})$

$\mathrm{ILCR}=\mathrm{LADD} \times \mathrm{CSF}$

$\mathrm{HQ}=\mathrm{LADD} / \mathrm{RfD}$

where, Cs is the pesticide concentration in vegetables $\left(\mathrm{mg} \mathrm{kg}^{-1}\right)$, IR is the vegetable intake rate $\left(137 \mathrm{~g} \mathrm{~d}^{-1}\right.$, single or mixed with others), $\mathrm{F}$ is the unit conversion factor, $\mathrm{EF}$ is exposure frequency (365 days/year), ED is the life time exposure duration (70 years), BW is the body weight $(70 \mathrm{~kg})$, and AT is the averaging time for carcinogens (EF $\times \mathrm{ED})$. $\mathrm{CSF}$ and $\mathrm{RfD}$ is oral cancer slope factor and reference dose respectively, for individual compound $\left(\mathrm{mg} \mathrm{kg}^{-1} \mathrm{~d}^{-1}\right){ }^{43}$

\section{Results}

\section{Residue levels}

\section{of hexachlorocyclohexane and dichlorodiphenyltrichloroethane}

Concentration means and ranges of $\mathrm{HCH}$ and DDT residues in different vegetables have been given in Table 1. In this study we studied the two major isomers of $\mathrm{HCH}(\alpha-\mathrm{HCH}$ and $\gamma$ $\mathrm{HCH}$ ). $\alpha-\mathrm{HCH}$ is main component of technical $\mathrm{HCH}$ while $\gamma$-HCH is the major component of lindane formulation. The observed level of $\alpha$ $\mathrm{HCH}$ and $\gamma$-HCH was ranged between 0.05-1.55 
$\mu \mathrm{gg}^{-1}$ and $0.06-6.61 \mu \mathrm{gg}^{-1}$ and their mean was 0.56 and $1.37, \mu \mathrm{g} \mathrm{kg}^{-1}$ respectively. The average concentrations of DDT isomers was $0.60 \mu \mathrm{gg}^{-1}, 1.02 \mu \mathrm{g} \mathrm{kg}^{-1}, 1.09 \mu \mathrm{g} \mathrm{kg}{ }^{-1}$ and 0.30 $\mu \mathrm{g} \mathrm{kg}^{-1}$, respectively for and $p, p^{\prime}$-DDT, $o, p^{\prime}$ DDT, $p, p$ '-DDE and $p, p$ '-DDD.

\section{Human health risk estimates}

Human health risk assessment was based on assumption that human being exposed to $\mathrm{HCH}$ and DDT pesticides through vegetables may cause harmful effects on humans. Human health risk due to exposure to HCHs and DDTs through vegetables was assessed, considering that humans take vegetables all the days in a year during the life span of 70 years. Risk was assessed by estimating the incremental LADD and subsequently their ILCR. The life time average daily dose is the amount of pollutant intake per kg of body weight per day, which may cause adverse health effects when absorbed into the body over a long period of time. The comparative LADD, ILCR and HI for humans from HCH and DDT exposure through studied vegetables has been shown in Figure 1.

Estimated LADD of individual HCH and DDT isomers and their total (HCHs+DDTs) was presented in Table 2. The estimated LADD of total pesticides from carrot, cauliflower, eggplant, radish, spinach and tomato was ranged between $8.5 \times 10^{-8} 2.1 \times 10^{-6} \mathrm{mg} \mathrm{kg}^{-1} \mathrm{~d}^{-1}, 5.0 \times 10^{-8} 4.3 \times 10^{-6}$ $\mathrm{mg} \mathrm{kg} \mathrm{kg}^{-1} \mathrm{~d}^{-1}, 3.1 \times 10^{-7} 1.3 \times 10^{-6} \mathrm{mg} \mathrm{kg}^{-1} \mathrm{~d}^{-1}$, $7.2 \times 10^{-7} 9.1 \times 10^{-6} \mathrm{mg} \mathrm{kg}^{-1} \mathrm{~d}^{-1}, 6.8 \times 10^{-8} 2.0 \times 10^{-6}$ $\mathrm{mg} \mathrm{kg} \mathrm{d}^{-1}$ and $4.1 \times 10^{-7} 2.9 \times 10^{-6} \mathrm{mg} \mathrm{kg}^{-1} \mathrm{~d}^{-1}$, respectively. The average LADD was $1.1 \times 10^{-6}$ $\mathrm{mg} \mathrm{kg}^{-1} \mathrm{~d}^{-1}, 2.5 \times 10^{-6} \mathrm{mg} \mathrm{kg}^{-1} \mathrm{~d}^{-1}, 7.8 \times 10^{-7} \mathrm{mg}$ $\mathrm{kg}^{-1} \mathrm{~d}^{-1}, 3.4 \times 10^{-6} \mathrm{mg} \mathrm{kg}^{-1} \mathrm{~d}^{-1}, 1.0 \times 10^{-6} \mathrm{mg} \mathrm{kg}^{-1} \mathrm{~d}^{-1}$ and $1.6 \times 10^{-6} \mathrm{mg} \mathrm{kg}^{-1} \mathrm{~d}^{-1}$, respectively from carrot, cauliflower, eggplant, radish, spinach and tomato.

Human health risk in terms of ILCR from HCHs and DDTs in vegetables has been estimated and presented in Table 3 . Total ILCR was varied between $2.7 \times 10^{-8} 2.6 \times 10^{-6}$ (mean, $1.2 \times 10^{-6}$ ), $1.7 \times 10^{-8} 5.3 \times 10^{-6}$ (mean, $2.9 \times 10^{-6}$ ), $2.8 \times 10^{-7} 2.2 \times 10^{-6} \quad$ (mean, $\left.1.3 \times 10^{-6}\right), 2.3 \times$ $10^{-7} 8.8 \times 10^{-6} \quad\left(3.9 \times 10^{-6}\right), 2.1 \times 10^{-8} 1.6 \times 10^{-6}$ (mean, $1.0 \times 10^{-6}$ ) and $6.5 \times 10^{-7} 5.2 \times 10^{-6}($ mean, $\left.2.8 \times 10^{-6}\right)$, respectively from carrot, cauliflower, eggplant, radish, spinach and tomato intakes.

Table 1. Concentrations of hexachlorocyclohexane and dichlorodiphenyltrichloroethane pesticides in different vegetables ( $\mu \mathrm{g} \mathrm{kg}^{-1}$ ).

\begin{tabular}{|c|c|c|c|c|c|c|c|c|c|c|}
\hline \multirow{2}{*}{$\begin{array}{l}\text { Vegetables } \\
\text { (no.)* }\end{array}$} & \multirow[b]{2}{*}{$\begin{array}{l}\alpha- \\
\mathrm{HCH}\end{array}$} & \multicolumn{6}{|c|}{ Pesticides compound concentrations } & \multicolumn{3}{|c|}{ Isomeric ratio } \\
\hline & & $\stackrel{\gamma-}{\mathrm{HCH}}$ & $\begin{array}{l}p, p^{\prime}- \\
\text { DDT }\end{array}$ & $\begin{array}{l}o, p^{\prime}- \\
\text { DDT }\end{array}$ & $\begin{array}{l}p, p^{\prime}- \\
\text { DDE }\end{array}$ & $\begin{array}{l}p, p^{\prime}- \\
\text { DDD }\end{array}$ & $\sum 0 \mathrm{CPs}$ & $\begin{array}{l}\alpha / \gamma- \\
\mathrm{HCH}\end{array}$ & $\begin{array}{c}o, p^{\prime} / p, p^{\prime}- \\
\text { DDT }\end{array}$ & $\begin{array}{c}\text { DDT/ } \\
\text { (DDE+DDE) }\end{array}$ \\
\hline $\begin{array}{l}\text { Carrot } \\
\text { (12) }\end{array}$ & $\begin{array}{c}0.31-0.64 \\
(0.48)^{\circ}\end{array}$ & $\begin{array}{c}0.59-0.84 \\
(0.72)\end{array}$ & $\begin{array}{c}0.13-0.28 \\
(0.19)\end{array}$ & $\begin{array}{c}0.13-3.14 \\
(1.23)\end{array}$ & $\begin{array}{c}0.10-0.19 \\
(0.15)\end{array}$ & $\begin{array}{c}0.04-1.19 \\
(0.39)\end{array}$ & $\begin{array}{l}0.18-4.46 \\
(2.18)\end{array}$ & $\begin{array}{c}0.53-0.76 \\
(0.64)\end{array}$ & $\begin{array}{c}0.55-2.38 \\
(1.49)\end{array}$ & $\begin{array}{c}1.57-1.88 \\
(1.23)\end{array}$ \\
\hline $\begin{array}{l}\text { Cauliflower } \\
\text { (13) }\end{array}$ & $\begin{array}{c}0.09-1.24 \\
(0.56) \\
\end{array}$ & $\begin{array}{c}0.18-5.34 \\
(1.73)\end{array}$ & $\begin{array}{c}0.43-1.68 \\
(0.97) \\
\end{array}$ & $\begin{array}{c}0.13-0.43 \\
(0.24) \\
\end{array}$ & $\begin{array}{c}0.10-6.29 \\
(2.01) \\
\end{array}$ & $\begin{array}{c}0.05-0.16 \\
(0.11)\end{array}$ & $\begin{array}{c}0.10-8.99 \\
(5.22) \\
\end{array}$ & $\begin{array}{c}0.09-3.37 \\
(0.73)\end{array}$ & $\begin{array}{c}0.08-0.57 \\
(0.27) \\
\end{array}$ & $\begin{array}{c}0.21-3.31 \\
(1.52) \\
\end{array}$ \\
\hline $\begin{array}{l}\text { Eggplant } \\
(9)\end{array}$ & $\begin{array}{c}0.05-0.62 \\
(0.25)\end{array}$ & $\begin{array}{c}0.06-2.24 \\
(0.77)\end{array}$ & $\begin{array}{c}0.12-0.23 \\
(0.17)\end{array}$ & $\begin{array}{c}0.09-0.31 \\
(0.18)\end{array}$ & $\begin{array}{c}0.04-0.63 \\
(0.20)\end{array}$ & $\begin{array}{c}0.04-0.08 \\
(0.07)\end{array}$ & $\begin{array}{c}0.65-2.77 \\
(1.63)\end{array}$ & $\begin{array}{c}0.09-2.12 \\
(0.84)\end{array}$ & $\begin{array}{c}0.69-2.50 \\
(1.18)\end{array}$ & $\begin{array}{c}0.52-3.98 \\
(3.11)\end{array}$ \\
\hline $\begin{array}{l}\text { Radish } \\
(7)\end{array}$ & $\begin{array}{c}0.10-1.53 \\
(0.94)\end{array}$ & $\begin{array}{c}0.20-6.61 \\
(1.88)\end{array}$ & $\begin{array}{c}0.15-1.82 \\
(0.85)\end{array}$ & $\begin{array}{c}0.86-11.08 \\
(3.76)\end{array}$ & $\begin{array}{c}0.07-3.28 \\
(1.04)\end{array}$ & $\begin{array}{c}0.08-2.92 \\
(0.95)\end{array}$ & $\begin{array}{c}1.50-18.81 \\
(7.00)\end{array}$ & $\begin{array}{c}0.23-2.46 \\
(1.15)\end{array}$ & $\begin{array}{c}0.68-2.83 \\
(1.88)\end{array}$ & $\begin{array}{c}1.20-2.57 \\
(1.75)\end{array}$ \\
\hline $\begin{array}{l}\text { Spinach } \\
\text { (15) }\end{array}$ & $\begin{array}{c}0.05-0.24 \\
(0.18)\end{array}$ & $\begin{array}{c}0.69-1.15 \\
(0.99)\end{array}$ & $\begin{array}{c}0.09-0.19 \\
(0.13)\end{array}$ & $\begin{array}{l}0.11-1.60 \\
(0.51)\end{array}$ & $\begin{array}{c}0.45-1.06 \\
(0.69)\end{array}$ & $\begin{array}{c}0.03-0.06 \\
(0.05)\end{array}$ & $\begin{array}{c}0.14-4.12 \\
(2.12)\end{array}$ & $\begin{array}{c}0.05-0.33 \\
(0.19)\end{array}$ & $\begin{array}{c}1.22-2.95 \\
(2.08)\end{array}$ & $\begin{array}{c}0.26-1.55 \\
(1.04)\end{array}$ \\
\hline $\begin{array}{l}\text { Tomato } \\
(8)\end{array}$ & $\begin{array}{c}0.06-1.55 \\
(0.67)\end{array}$ & $\begin{array}{c}0.09-3.64 \\
(1.04)\end{array}$ & $\begin{array}{c}0.12-1.71 \\
(0.46)\end{array}$ & $\begin{array}{c}0.09-1.69 \\
(0.87)\end{array}$ & $\begin{array}{c}0.04-0.22 \\
(0.11)\end{array}$ & $\begin{array}{c}0.03-0.93 \\
(0.27)\end{array}$ & $\begin{array}{c}0.84-5.91 \\
(3.25)\end{array}$ & $\begin{array}{c}0.04-5.69 \\
(1.70)\end{array}$ & $\begin{array}{c}0.65-0.99 \\
(0.79)\end{array}$ & $\begin{array}{c}1.45-3.45 \\
(2.52)\end{array}$ \\
\hline All samples (64) & $\begin{array}{c}0.05-1.55 \\
(0.56)\end{array}$ & $\begin{array}{c}0.06-6.61 \\
(1.37)\end{array}$ & $\begin{array}{c}0.09-1.82 \\
(0.60)\end{array}$ & $\begin{array}{c}0.09-11.08 \\
(1.02)\end{array}$ & $\begin{array}{c}0.04-6.29 \\
(1.09)\end{array}$ & $\begin{array}{c}0.03-2.92 \\
(0.30)\end{array}$ & $\begin{array}{c}0.10-18.81 \\
(4.18)\end{array}$ & $\begin{array}{c}0.04-5.69 \\
(0.86)\end{array}$ & $\begin{array}{c}0.08-2.95 \\
(0.82)\end{array}$ & $\begin{array}{c}0.21-3.98 \\
(1.77)\end{array}$ \\
\hline
\end{tabular}

*Number of samples, ${ }^{\circ}$ average in parenthesis. HCH, hexachlorocyclohexane; DDT, dichlorodiphenyltrichloroethane; OCP, organochlorine pesticides; DDE, dichlorodiphenyldichloroethylene.

Table 2. Estimated average daily dose $\left(\mathrm{mg} \mathrm{kg}^{-1} \mathrm{~d}^{-1}\right)$ of hexachlorocyclohexane and dichlorodiphenyltrichloroethane through vegetable to humans.

\begin{tabular}{|c|c|c|c|c|c|c|c|}
\hline \multirow{2}{*}{$\begin{array}{l}\text { Vegetables } \\
\text { (no.)* }\end{array}$} & \multirow[b]{2}{*}{$\alpha-\mathrm{HCH}$} & \multicolumn{5}{|c|}{ ADD of pesticides compounds } & \multirow[t]{2}{*}{ Total ADD } \\
\hline & & $\gamma$-HCH & $p, p^{\prime}-\mathrm{DDT}$ & $0, p^{\prime}-\mathrm{DDT}$ & $p, p^{\prime}-\mathrm{DDE}$ & $p, p^{\prime}-\mathrm{DDD}$ & \\
\hline $\begin{array}{l}\text { Carrot } \\
(12)\end{array}$ & $\begin{array}{c}1.5 \times 10^{-7}-3.1 \times 10^{-7} \\
\left(2.3 \times 10^{-7}\right)^{\circ}\end{array}$ & $\begin{array}{c}2.8 \times 10^{-7}-4.1 \times 10^{-7} \\
\left(3.4 \times 10^{-7}\right)\end{array}$ & $\begin{array}{c}6.3 \times 10^{-8}-1.3 \times 10^{-7} \\
\left(9.3 \times 10^{-8}\right)\end{array}$ & $\begin{array}{c}6.2 \times 10^{-8}-1.5 \times 10^{-6} \\
\left(5.9 \times 10^{-7}\right)\end{array}$ & $\begin{array}{c}5.0 \times 10^{-8}-9.2 \times 10^{-8} \\
\left(7.1 \times 10^{-8}\right)\end{array}$ & $\begin{array}{c}2.1 \times 10^{-8}-5.7 \times 10^{-7} \\
\left(1.9 \times 10^{-7}\right)\end{array}$ & $\begin{array}{c}8.5 \times 10^{-8}-2.1 \times 10^{-6} \\
\left(1.1 \times 10^{-6}\right)\end{array}$ \\
\hline $\begin{array}{l}\text { Cauliflower } \\
\text { (13) }\end{array}$ & $\begin{array}{c}4.4 \times 10^{-8}-6.0 \times 10^{-7} \\
\left(2.7 \times 10^{-7}\right)\end{array}$ & $\begin{array}{c}8.4 \times 10^{-8}-2.6 \times 10^{-6} \\
\left(8.3 \times 10^{-7}\right)\end{array}$ & 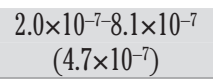 & $\begin{array}{c}6.3 \times 10^{-8}-2.1 \times 10^{-7} \\
\left(1.2 \times 10^{-7}\right)\end{array}$ & $\begin{array}{c}5.0 \times 10^{-8}-3.0 \times 10^{-6} \\
\left(9.7 \times 10^{-7}\right)\end{array}$ & $\begin{array}{c}2.5 \times 10^{-8}-7.6 \times 10^{-8} \\
\left(5.4 \times 10^{-8}\right)\end{array}$ & $\begin{array}{c}5.0 \times 10^{-8}-4.3 \times 10^{-6} \\
\left(2.5 \times 10^{-6}\right)\end{array}$ \\
\hline $\begin{array}{l}\text { Eggplant } \\
(9)\end{array}$ & $\begin{array}{c}2.6 \times 10^{-8}-3.0 \times 10^{-7} \\
\left(1.2 \times 10^{-7}\right)\end{array}$ & $\begin{array}{c}2.9 \times 10^{-8}-1.1 \times 10^{-6} \\
\left(3.7 \times 10^{-7}\right)\end{array}$ & $\begin{array}{c}5.5 \times 10^{-8}-1.1 \times 10^{-7} \\
\left(8.0 \times 10^{-8}\right)\end{array}$ & $\begin{array}{c}4.2 \times 10^{-8}-1.5 \times 10^{-7} \\
\left(8.5 \times 10^{-8}\right)\end{array}$ & $\begin{array}{c}1.9 \times 10^{-8}-3.0 \times 10^{-7} \\
\left(9.7 \times 10^{-8}\right)\end{array}$ & $\begin{array}{c}1.8 \times 10^{-8}-4.0 \times 10^{-8} \\
\left(3.2 \times 10^{-8}\right)\end{array}$ & $\begin{array}{c}3.1 \times 10^{-7}-1.3 \times 10^{-6} \\
\left(7.8 \times 10^{-7}\right)\end{array}$ \\
\hline $\begin{array}{l}\text { Radish } \\
\text { (7) }\end{array}$ & $\begin{array}{c}4.8 \times 10^{-8}-7.4 \times 10^{-7} \\
\left(4.5 \times 10^{-7}\right)\end{array}$ & $\begin{array}{c}9.8 \times 10^{-8}-3.2 \times 10^{-6} \\
\left(9.1 \times 10^{-7}\right)\end{array}$ & $\begin{array}{c}7.1 \times 10^{-8}-8.8 \times 10^{-7} \\
\left(4.1 \times 10^{-7}\right)\end{array}$ & $\begin{array}{c}4.2 \times 10^{-7}-5.3 \times 10^{-6} \\
\left(1.8 \times 10^{-6}\right)\end{array}$ & $\begin{array}{c}3.2 \times 10^{-8}-1.6 \times 10^{-6} \\
\left(5.0 \times 10^{-7}\right)\end{array}$ & $\begin{array}{c}3.8 \times 10^{-8}-1.4 \times 10^{-6} \\
\left(4.6 \times 10^{-7}\right)\end{array}$ & $\begin{array}{c}7.2 \times 10^{-7}-9.1 \times 10^{-6} \\
\left(3.4 \times 10^{-6}\right)\end{array}$ \\
\hline $\begin{array}{l}\text { Spinach } \\
\text { (15) }\end{array}$ & $\begin{array}{c}2.6 \times 10^{-8}-1.1 \times 10^{-7} \\
\left(8.6 \times 10^{-8}\right)\end{array}$ & $\begin{array}{c}3.3 \times 10^{-7}-5.5 \times 10^{-7} \\
\left(4.8 \times 10^{-7}\right)\end{array}$ & $\begin{array}{c}4.4 \times 10^{-8}-9.2 \times 10^{-8} \\
\left(6.5 \times 10^{-8}\right)\end{array}$ & $\begin{array}{c}5.2 \times 10^{-8}-7.7 \times 10^{-7} \\
\left(2.5 \times 10^{-7}\right)\end{array}$ & $\begin{array}{c}2.1 \times 10^{-7}-5.1 \times 10^{-7} \\
\left(3.3 \times 10^{-7}\right)\end{array}$ & $\begin{array}{c}1.6 \times 10^{-8}-2.9 \times 10^{-8} \\
\left(2.2 \times 10^{-8}\right)\end{array}$ & $\begin{array}{c}6.8 \times 10^{-8}-2.0 \times 10^{-6} \\
\left(1.0 \times 10^{-6}\right)\end{array}$ \\
\hline $\begin{array}{l}\text { Tomato } \\
\text { (8) }\end{array}$ & $\begin{array}{c}2.8 \times 10^{-8-7} .5 \times 10^{-7} \\
\left(3.2 \times 10^{-7}\right)\end{array}$ & $\begin{array}{c}4.3 \times 10^{-8}-1.8 \times 10^{-6} \\
\left(5.0 \times 10^{-7}\right)\end{array}$ & $\begin{array}{c}5.9 \times 10^{-8}-8.2 \times 10^{-7} \\
\left(2.2 \times 10^{-7}\right)\end{array}$ & $\begin{array}{c}4.5 \times 10^{-8}-8.1 \times 10^{-7} \\
\left(4.2 \times 10^{-7}\right)\end{array}$ & $\begin{array}{c}2.1 \times 10^{-8}-1.1 \times 10^{-7} \\
\left(5.5 \times 10^{-8}\right)\end{array}$ & $\begin{array}{c}1.4 \times 10^{-8}-4.5 \times 10^{-7} \\
\left(1.3 \times 10^{-7}\right)\end{array}$ & $\begin{array}{c}4.1 \times 10^{-7}-2.9 \times 10^{-6} \\
\left(1.6 \times 10^{-6}\right)\end{array}$ \\
\hline $\begin{array}{l}\text { All samples } \\
\text { (64) }\end{array}$ & $\begin{array}{c}2.6 \times 10^{-8-7.5 \times 10^{-7}} \\
\left(2.7 \times 10^{-7}\right)\end{array}$ & $\begin{array}{c}2.9 \times 10^{-8}-3.2 \times 10^{-6} \\
\left(6.6 \times 10^{-7}\right)\end{array}$ & $\begin{array}{c}4.4 \times 10^{-8}-8.8 \times 10^{-7} \\
\left(2.9 \times 10^{-7}\right)\end{array}$ & $\begin{array}{c}4.2 \times 10^{-8}-5.3 \times 10^{-6} \\
\left(4.9 \times 10^{-7}\right)\end{array}$ & $\begin{array}{c}1.9 \times 10^{-8}-3.0 \times 10^{-6} \\
\left(5.2 \times 10^{-7}\right)\end{array}$ & $\begin{array}{c}1.4 \times 10^{-8}-1.4 \times 10^{-6} \\
\left(1.4 \times 10^{-7}\right)\end{array}$ & $\begin{array}{c}5.0 \times 10^{-8}-9.1 \times 10^{-6} \\
\left(2.0 \times 10^{-6}\right)\end{array}$ \\
\hline
\end{tabular}

*Number of samples; 'average in parenthesis. ADD, average daily dose; HCH, hexachlorocyclohexane; DDT, dichlorodiphenyltrichloroethane; DDE, dichlorodiphenyldichloroethylene; DDD, dichlorodiphenyldichloroethane. 


\section{Discussion}

\section{Possible sources identification}

HCH formulations are available as technical $\mathrm{HCH}$ and lindane. Technical HCH contains 55$80 \%$ and $8-15 \% \alpha-\mathrm{HCH}$ and $\gamma-\mathrm{HCH}$ respectively, while lindane contains $>90 \%$ of $\gamma-\mathrm{HCH}$. Therefore, ratio of $\alpha-\mathrm{HCH}$ to $\gamma-\mathrm{HCH}$ has been used for identification of possible $\mathrm{HCH}$ sources. The ratio of $\alpha-\mathrm{HCH}$ to $\gamma-\mathrm{HCH}$ between 3 and 7 infer the input of technical HCH. ${ }^{44}$ However, lindane source are characterized by reduced ratio ( $\leq 1)$. A higher ratio $(>7)$ of $\alpha-\mathrm{HCH}$ to $\gamma-\mathrm{HCH}$ was explained by long-range transport or recycling of technical $\mathrm{HCH}$, because $\alpha-\mathrm{HCH}$ has a longer atmospheric lifetime than $\gamma$ - $\mathrm{HCH}$ by about $25 \%{ }^{45} \gamma$-HCH may be transformed by sunlight and through biological degradation into $\alpha$ HCH. ${ }^{46,47}$ The $\alpha-\mathrm{HCH}$ to $\gamma-\mathrm{HCH}$ isomers ratio ( $\alpha / \gamma$ ratio) in this study was ranged between 0.04 to 5.69 with a mean of 0.82 which reflect the local input of technical $\mathrm{HCH}$ as well as lindane formulation. The usage of technical $\mathrm{HCH}$ and lindane has been banned in agriculture but, used in public health programmes to control vector borne diseases. ${ }^{6}$

The pattern of individual DDT isomers may presume the hypothesis of DDT sources. ${ }^{48}$ Three major isomers (DDT, DDD and DDE) have been used for assessing the chronology of DDT input. Changes in the ratio between DDE and DDD to DDTs have been regarded as an indication of either no, or decreasing, inputs of DDT. Ratio of DDT/(DDD+DDE) greater than 1 indicates past DDT inputs in last five years, and lower ratios suggests aged DDT sources (possibly microbial degradation). ${ }^{49,50}$ The average ratio of DDT/(DDD+DDE) for this study was 1.77, indicating past usage of DDT. Generally, technical grade DDT constitute $77.1 \%$ ( $p, p^{\prime}$-DDT), $14.9 \%$ (o,p'-DDT), $4.0 \%$ (p,p'-DDE), $0.3 \%$ ( $p, p^{\prime}$-DDD), and $0.1 \%\left(o, p^{\prime}\right.$-DDD).$^{51}$ However, in this study we observed that $p, p^{\prime}$-DDE and $o, p^{\prime}$-DDT were the dominant isomers. The vapour pressure of $o, p$ '-DDT is 7.5 times higher than $p, p^{\prime}$-DDT leading to greater volatilization of $o, p^{\prime}$-DDT to the atmosphere and $p, p^{\prime}$-DDT metabolizes much faster in a tropical environment. ${ }^{50}$ DDT is known to biodegrade to DDE under aerobic and to DDD under anaerobic conditions. DDE, the most prevalent breakdown product of DDT is persistent in the environment and bioaccumulates in the food chain thereby gets stored in fatty tissues of animals, fish, and humans..$^{52}$ In the environment, much of the DDT might have been converted to $p, p$ '-DDE. Elevated levels of $p, p$ 'DDE have been interpreted as a result of its con- version to $p, p^{\prime}$-DDE by UV radiation. ${ }^{53}$ The $o, p^{\prime}$ $\mathrm{DDT} / p, p^{\prime}$-DDT ratio reported to be $0.2 \sim 0.26$ in DDT and 7.5 in dicofol products. ${ }^{54}$ The ratio of $o, p^{\prime}$-DDT $/ p, p^{\prime}$-DDT in the studied vegetables ranged from 0.08 to 2.95 with the mean of 0.82 . This average ratio of $o, p^{\prime}$-DDT/ $p, p^{\prime}$-DDT lower than that of dicofol but higher than that of DDT. These observed ratios of DDT/(DDD+DDE) and $o, p^{\prime}$-DDT/ $p, p^{\prime}$-DDT suggest combined contamination effect from past and ongoing usage of DDT coupled with the long-range atmospheric transport tendency of DDT under tropical climate conditions but not from dicofol. DDT usage has been banned in agriculture, ${ }^{55}$ however, India has permission for limited DDT production and use of residual spray in public health practices for vector control. ${ }^{56}$ Concentration of total pesticides (HCHs+DDTs) in all vegetable samples

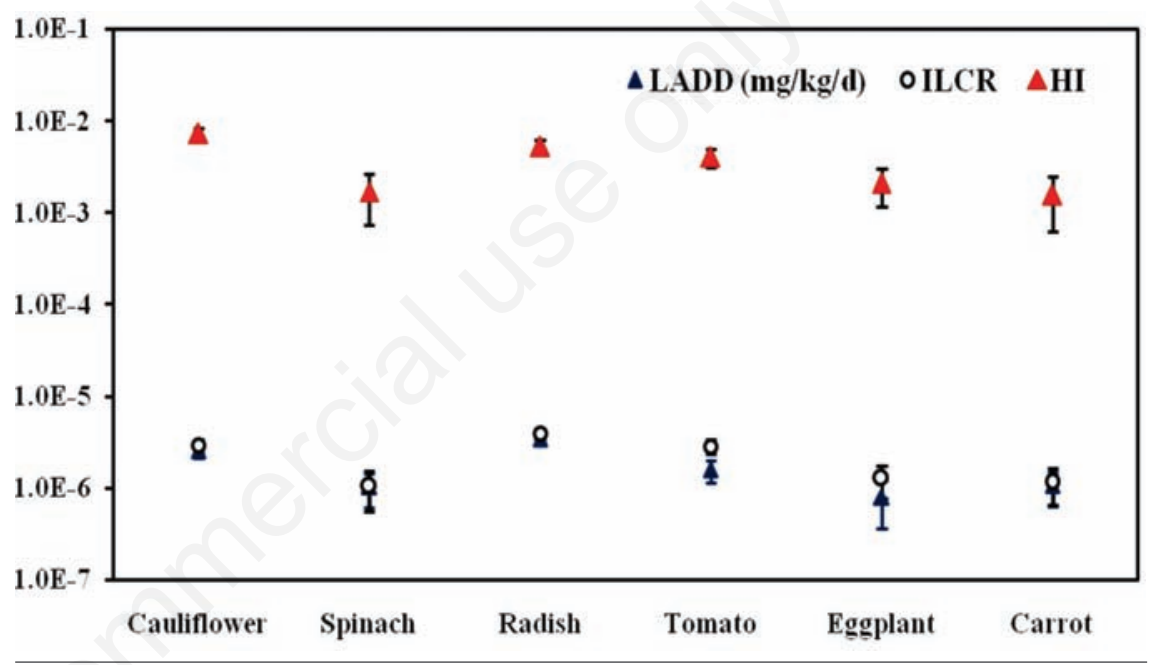

Figure 1. Comparative lifetime average daily dose (LADD), incremental lifetime cancer risk (ILCR) and hazard index (HI) for selected humans due to hexachlorocyclohexane and dichlorodiphenyltrichloroethane exposure through vegetables.

Table 3. Probable incremental life time cancer risk to humans from hexachlorocyclohexane and dichlorodiphenyltrichloroethane through vegetables.

\begin{tabular}{|c|c|c|c|c|c|c|c|}
\hline \multirow{2}{*}{$\begin{array}{l}\text { Vegetables } \\
\text { (no.)* }\end{array}$} & \multirow[b]{2}{*}{$\alpha-\mathrm{HCH}$} & \multicolumn{5}{|c|}{ Risk of pesticides compounds } & \multirow[t]{2}{*}{ Total risk } \\
\hline & & $\gamma$-HCH & $p, p^{\prime}-\mathrm{DDT}$ & & $p, p^{\prime}-\mathrm{DDE}$ & $p, p^{\prime}-\mathrm{DDD}$ & \\
\hline $\begin{array}{l}\text { arrot } \\
12)\end{array}$ & $\begin{array}{c}9.5 \times 10^{-7}-1.9 \times 10^{-6} \\
\left(1.4 \times 10^{-6}\right)^{\circ}\end{array}$ & $\begin{array}{c}3.1 \times 10^{-7}-4.5 \times 10^{-7} \\
\left(3.8 \times 10^{-7}\right)\end{array}$ & $\begin{array}{c}2.1 \times 10^{-8}-4.6 \times 10^{-8} \\
\left(3.2 \times 10^{-8}\right)\end{array}$ & $\begin{array}{c}2.1 \times 10^{-8}-5.1 \times 10^{-7} \\
\left(2.0 \times 10^{-7}\right)\end{array}$ & $\begin{array}{c}1.7 \times 10^{-8}-3.1 \times 10^{-8} \\
\left(2.4 \times 10^{-8}\right)\end{array}$ & $\begin{array}{c}5.1 \times 10^{-9}-1.4 \times 10^{-7} \\
\left(4.6 \times 10^{-8}\right)\end{array}$ & $\begin{array}{l}.6 \times 10^{-6} \\
\left.0^{-6}\right)\end{array}$ \\
\hline $\begin{array}{l}\text { auliflower } \\
\text { 13) }\end{array}$ & $\begin{array}{c}2.8 \times 10^{-7}-3.8 \times 10^{-6} \\
\left(1.7 \times 10^{-6}\right)\end{array}$ & $\begin{array}{r}9.3 \times 10^{-8}-2 \\
(9.2 \times 1\end{array}$ & $\begin{array}{c}7.0 \times 10^{-8}-2.8 \times 10^{-7} \\
\left(1.6 \times 10^{-7}\right)\end{array}$ & $\begin{array}{c}2.1 \times 10^{-8}-7.0 \times 10^{-8} \\
\left(4.0 \times 10^{-8}\right)\end{array}$ & $\begin{array}{c}1.7 \times 10^{-8}-1.0 \times 10^{-6} \\
\left(3.3 \times 10^{-7}\right)\end{array}$ & $\begin{array}{c}6.0 \times 10^{-9}-1.8 \times 10^{-8} \\
\left(1.3 \times 10^{-8}\right)\end{array}$ & $\begin{array}{r}1.7 \times 1 \\
(2\end{array}$ \\
\hline $\begin{array}{l}\text { Eggplant } \\
(9)\end{array}$ & $\begin{array}{c}1.6 \times 10^{-7}-1.9 \times 10^{-6} \\
\left(7.6 \times 10^{-7}\right)\end{array}$ & $\begin{array}{c}3.2 \times 10^{-8}-1.2 \times 10^{-6} \\
\left(4.1 \times 10^{-7}\right)\end{array}$ & $\begin{array}{c}1.9 \times 10^{-8}-3.8 \times 10^{-8} \\
\left(2.7 \times 10^{-8}\right)\end{array}$ & $\begin{array}{c}1.4 \times 10^{-8}-5.0 \times 10^{-8} \\
\left(2.9 \times 10^{-8}\right)\end{array}$ & $\begin{array}{c}6.6 \times 10^{-9}-1.0 \times 10^{-7} \\
\left(3.3 \times 10^{-8}\right)\end{array}$ & $\begin{array}{c}4.3 \times 10^{-9}-9.6 \times 10-9 \\
(7.6 \times 10-9)\end{array}$ & $\begin{array}{l}2.2 \times 10^{-6} \\
\left.10^{-6}\right)\end{array}$ \\
\hline $\begin{array}{l}\text { Radish } \\
\text { (7) }\end{array}$ & $\begin{array}{c}3.0 \times 10^{-7}-4.6 \times 10^{-6} \\
\left(2.9 \times 10^{-6}\right)\end{array}$ & $\begin{array}{c}1.1 \times 10^{-7}-3.5 \times 10^{-6} \\
\left(1.0 \times 10^{-6}\right)\end{array}$ & $\begin{array}{c}2.4 \times 10^{-8}-3.0 \times 10^{-7} \\
\left(1.4 \times 10^{-7}\right)\end{array}$ & $\begin{array}{c}1.4 \times 10^{-7}-1.8 \times 10^{-6} \\
\left(6.2 \times 10^{-7}\right)\end{array}$ & $\begin{array}{c}1.1 \times 10^{-8}-5.4 \times 10^{-7} \\
\left(1.7 \times 10^{-7}\right)\end{array}$ & $\begin{array}{c}9.1 \times 10^{-9}-3.4 \times 10^{-7} \\
\left(1.1 \times 10^{-7}\right)\end{array}$ & $\begin{array}{c}2.3 \times 10^{-7}-8.8 \times 10^{-6} \\
\left(3.9 \times 10^{-6}\right)\end{array}$ \\
\hline $\begin{array}{l}\text { Spinach } \\
\text { (15) }\end{array}$ & $\begin{array}{c}1.6 \times 10^{-7}-7.2 \times 10^{-7} \\
\left(5.4 \times 10^{-7}\right)\end{array}$ & $\begin{array}{c}3.7 \times 10^{-7}-6.1 \times 10^{-7} \\
\left(5.2 \times 10^{-7}\right)\end{array}$ & $\begin{array}{c}1.5 \times 10^{-8}-3.1 \times 10^{-8} \\
\left(2.2 \times 10^{-8}\right)\end{array}$ & $\begin{array}{c}1.8 \times 10^{-8}-2.6 \times 10^{-7} \\
\left(8.3 \times 10^{-8}\right)\end{array}$ & $\begin{array}{c}7.3 \times 10^{-8}-1.7 \times 10^{-7} \\
\left(1.1 \times 10^{-7}\right)\end{array}$ & $\begin{array}{c}3.8 \times 10^{-9}-6.9 \times 10^{-9} \\
(5.2 \times 10-9)\end{array}$ & $\begin{array}{c}2.1 \times 10^{-8}-1.6 \times 10^{-6} \\
\left(1.0 \times 10^{-6}\right)\end{array}$ \\
\hline $\begin{array}{l}\text { Tomato } \\
(8)\end{array}$ & $\begin{array}{c}1.8 \times 10^{-7}-4.7 \times 10^{-6} \\
\left(2.0 \times 10^{-6}\right)\end{array}$ & $\begin{array}{c}4.8 \times 10^{-8}-1.9 \times 10^{-6} \\
\left(5.5 \times 10^{-7}\right)\end{array}$ & $\begin{array}{c}2.0 \times 10^{-8}-2.8 \times 10^{-7} \\
\left(7.5 \times 10^{-8}\right)\end{array}$ & $\begin{array}{c}1.5 \times 10^{-8}-2.8 \times 10^{-7} \\
\left(1.4 \times 10^{-7}\right)\end{array}$ & $\begin{array}{c}7.0 \times 10^{-9}-3.6 \times 10^{-8} \\
\left(1.9 \times 10^{-8}\right)\end{array}$ & $\begin{array}{c}3.5 \times 10^{-9}-1.1 \times 10^{-7} \\
\left(3.1 \times 10^{-8}\right)\end{array}$ & $\begin{array}{c}6.5 \times 10^{-7}-5.2 \times 10^{-6} \\
\left(2.8 \times 10^{-6}\right)\end{array}$ \\
\hline $\begin{array}{l}\text { ll samples } \\
\text { 64) }\end{array}$ & $\begin{array}{c}1.6 \times 10^{-7}-4.7 \times 10^{-6} \\
\left(1.7 \times 10^{-6}\right)\end{array}$ & $\begin{array}{c}3.2 \times 10^{-8}-3.5 \times 10^{-6} \\
\left(7.3 \times 10^{-7}\right)\end{array}$ & $\begin{array}{c}1.5 \times 10^{-8}-3.0 \times 10^{-7} \\
\left(9.8 \times 10^{-8}\right)\end{array}$ & $\begin{array}{c}1.4 \times 10^{-8}-1.8 \times 10^{-6} \\
\left(1.7 \times 10^{-7}\right)\end{array}$ & $\begin{array}{c}6.6 \times 10^{-9}-1.0 \times 10^{-6} \\
\left(1.8 \times 10^{-7}\right)\end{array}$ & $\begin{array}{c}3.5 \times 10-9-3.4 \times 10^{-7} \\
\left(3.5 \times 10^{-8}\right)\end{array}$ & $\begin{array}{c}1.7 \times 10^{-8}-8.8 \times 10^{-6} \\
\left(2.5 \times 10^{-6}\right)\end{array}$ \\
\hline
\end{tabular}

*Number of samples; ${ }^{\circ}$ average in parenthesis. HCH, hexachlorocyclohexane; DDT, dichlorodiphenyltrichloroethane; DDE, dichlorodiphenyldichloroethylene; DDD, dichlorodiphenyldichloroethane. 
Table 4. Non-carcinogenic health hazard and hazard index for humans from hexachlorocyclohexane and dichlorodiphenyltrichloroethane through vegetables.

\begin{tabular}{|c|c|c|c|c|c|c|c|}
\hline $\begin{array}{l}\text { Vegetables } \\
\text { (no.)* }\end{array}$ & $\alpha-\mathrm{HCH}$ & $\gamma$-HCH & $\begin{array}{c}\text {-cancer HQs of } \\
p, p '-D D T\end{array}$ & $\begin{array}{l}\text { sticide compou } \\
\text { o,p'-DDT }\end{array}$ & $p, p^{\prime}-\mathrm{DDE}$ & $p, p^{\prime}-\mathrm{DDD}$ & HI \\
\hline $\begin{array}{l}\text { Carrot } \\
\text { (12) }\end{array}$ & $\begin{array}{c}1.9 \times 10^{-5}-3.8 \times 10^{-5} \\
\left(2.9 \times 10^{-5}\right)\end{array}$ & $\begin{array}{c}9.5 \times 10^{-4}-1.4 \times 10^{-3} \\
\left(1.1 \times 10^{-3}\right)\end{array}$ & $\begin{array}{c}6.5 \times 10^{-4}-1.4 \times 10^{-3} \\
\left(9.6 \times 10^{-4}\right)\end{array}$ & $\begin{array}{c}1.8 \times 10^{-7}-4.5 \times 10^{-6} \\
\left(1.7 \times 10^{-6}\right)\end{array}$ & $-\#$ & $-\#$ & $\begin{array}{c}6.6 \times 10^{-4}-2.8 \times 10^{-3} \\
\left(1.5 \times 10^{-3}\right)\end{array}$ \\
\hline $\begin{array}{l}\text { Cauliflower } \\
\text { (13) }\end{array}$ & $\begin{array}{c}5.5 \times 10^{-6}-7.4 \times 10^{-5} \\
\left(3.4 \times 10^{-5}\right)\end{array}$ & $\begin{array}{c}2.8 \times 10^{-4}-8.6 \times 10^{-3} \\
\left(2.8 \times 10^{-3}\right)\end{array}$ & $\begin{array}{c}2.1 \times 10^{-3}-8.4 \times 10^{-3} \\
\left(4.8 \times 10^{-3}\right)\end{array}$ & $\begin{array}{c}1.8 \times 10^{-7}-6.1 \times 10^{-7} \\
\left(3.5 \times 10^{-7}\right)\end{array}$ & - & - & $\begin{array}{c}1.7 \times 10^{-3}-1.7 \times 10^{-2} \\
\left(7.2 \times 10^{-3}\right)\end{array}$ \\
\hline $\begin{array}{l}\text { Eggplant } \\
(9)\end{array}$ & $\begin{array}{c}3.3 \times 10^{-6}-3.7 \times 10^{-5} \\
\left(1.5 \times 10^{-5}\right)\end{array}$ & $\begin{array}{c}9.8 \times 10^{-5}-3.6 \times 10^{-3} \\
\left(1.2 \times 10^{-3}\right)\end{array}$ & $\begin{array}{c}5.7 \times 10^{-4}-1.1 \times 10^{-3} \\
\left(8.3 \times 10^{-4}\right)\end{array}$ & $\begin{array}{c}1.2 \times 10^{-7}-4.4 \times 10^{-7} \\
\left(2.5 \times 10^{-7}\right)\end{array}$ & - & - & $\begin{array}{c}7.1 \times 10^{-4}-4.2 \times 10^{-3} \\
\left(2.1 \times 10^{-3}\right)\end{array}$ \\
\hline $\begin{array}{l}\text { Radish } \\
\text { (7) }\end{array}$ & $\begin{array}{c}6.0 \times 10^{-6}-9.2 \times 10^{-5} \\
\left(5.7 \times 10^{-5}\right)\end{array}$ & $\begin{array}{c}3.3 \times 10^{-4}-1.1 \times 10^{-2} \\
\left(3.0 \times 10^{-3}\right)\end{array}$ & $\begin{array}{c}7.3 \times 10^{-4}-9.0 \times 10^{-3} \\
\left(4.2 \times 10^{-3}\right)\end{array}$ & $\begin{array}{c}1.2 \times 10^{-6}-1.6 \times 10^{-5} \\
\left(5.3 \times 10^{-6}\right)\end{array}$ & - & - & $\begin{array}{c}8.9 \times 10^{-5}-1.1 \times 10^{-2} \\
\left(5.2 \times 10^{-3}\right)\end{array}$ \\
\hline $\begin{array}{l}\text { Spinach } \\
\text { (15) }\end{array}$ & $\begin{array}{c}3.2 \times 10^{-6}-1.4 \times 10^{-5} \\
\left(1.1 \times 10^{-5}\right)\end{array}$ & $\begin{array}{c}1.1 \times 10^{-3}-1.8 \times 10^{-3} \\
\left(1.6 \times 10^{-3}\right)\end{array}$ & $\begin{array}{c}4.6 \times 10^{-4}-9.4 \times 10^{-4} \\
\left(4.0 \times 10^{-4}\right)\end{array}$ & $\begin{array}{c}1.5 \times 10^{-7}-2.3 \times 10^{-6} \\
\left(7.2 \times 10^{-7}\right)\end{array}$ & - & - & $\begin{array}{c}1.5 \times 10^{-7}-2.4 \times 10^{-3} \\
\left(1.7 \times 10^{-3}\right)\end{array}$ \\
\hline $\begin{array}{l}\text { Tomato } \\
(8)\end{array}$ & $\begin{array}{c}3.6 \times 10^{-6}-9.4 \times 10^{-5} \\
\left(4.0 \times 10^{-5}\right)\end{array}$ & $\begin{array}{c}1.4 \times 10^{-4}-5.9 \times 10^{-3} \\
\left(1.7 \times 10^{-3}\right)\end{array}$ & $\begin{array}{c}6.1 \times 10^{-4}-8.5 \times 10^{-3} \\
\left(2.3 \times 10^{-3}\right)\end{array}$ & $\begin{array}{c}1.3 \times 10^{-7}-2.4 \times 10^{-6} \\
\left(1.2 \times 10^{-6}\right)\end{array}$ & - & - & $\begin{array}{c}1.1 \times 10^{-3}-8.7 \times 10^{-3} \\
\left(4.0 \times 10^{-3}\right)\end{array}$ \\
\hline $\begin{array}{l}\text { All samples } \\
(64)\end{array}$ & $\begin{array}{c}3.2 \times 10^{-6}-9.4 \times 10^{-5} \\
\left(3.4 \times 10^{-5}\right)^{\circ}\end{array}$ & $\begin{array}{c}9.8 \times 10^{-5}-1.1 \times 10^{-2} \\
\left(2.2 \times 10^{-3}\right)\end{array}$ & $\begin{array}{c}4.6 \times 10^{-4}-9.0 \times 10^{-3} \\
\left(3.0 \times 10^{-3}\right)\end{array}$ & $\begin{array}{c}1.2 \times 10^{-7}-1.6 \times 10^{-5} \\
\left(1.4 \times 10^{-6}\right)\end{array}$ & - & - & $\begin{array}{c}1.5 \times 10^{-7}-1.7 \times 10^{-2} \\
\left(4.5 \times 10^{-3}\right)\end{array}$ \\
\hline
\end{tabular}

*Number of samples; ${ }^{\circ}$ average in parenthesis; ${ }^{*}$ not calculated-no reference dose assigned. HQ, health hazard; HI, hazard index; HCH, hexachlorocyclohexane; DDT, dichlorodiphenyltrichloroethane; DDE, dichlorodiphenyldichloroethylene; DDD, dichlorodiphenyldichloroethane.

was ranged from $0.04 \mu \mathrm{gg} \mathrm{kg}^{-1}$ to $5.69 \mu \mathrm{g} \mathrm{kg} \mathrm{kg}^{-1}$ with the mean value of $4.18 \mu \mathrm{gg}^{-1}$. The observed levels of $\mathrm{HCH}$ and DDT in vegetables were compared with maximum residual levels (MRLs) and found lower than the recommended values from European countries and Indian government. ${ }^{57,58}$

\section{Human health risk}

The overall LADD for all studied vegetables was ranges between $5.0 \times 10^{-8} 9.1 \times 10^{-6} \mathrm{mg} \mathrm{kg}^{-1}$ $\mathrm{d}^{-1}$ with an average value of $2.0 \times 10^{-6} \mathrm{mg} \mathrm{kg}^{-1}$ $\mathrm{d}^{-1}$. These estimated average daily intakes were within recommended acceptable limits of reference dose (RfD) for $\mathrm{HCHs}(\alpha-\mathrm{HCH}$, $\left.8 \times 10^{-3} \mathrm{mg} \mathrm{kg}^{-1} \mathrm{~d}^{-1} ; \gamma-\mathrm{HCH}, 3 \times 10^{-3} \mathrm{mg} \mathrm{kg}^{-1} \mathrm{~d}^{-1}\right)$ and DDTs $\left(o, p^{\prime}\right.$-DDT, $5 \times 10^{-4} \mathrm{mg} \mathrm{kg}^{-1} \mathrm{~d}^{-1} ; p, p^{\prime}-$ DDT, $\left.9.7 \times 10^{-5} \mathrm{mg} \mathrm{kg}^{-1} \mathrm{~d}^{-1}\right)$. ${ }^{43,59}$ ILCR for all the samples was ranges between $1.7 \times 10^{-8} 8.8 \times 10^{-6}$ with an average of $2.5 \times 10^{-6}$. This estimated ILCR was within the acceptable excess risk distribution range $\left(10^{-6} 10^{-4}\right) .{ }^{43}$ Acceptable limits of excess cancer risks recommended by United States Environmental Protection Agency are ranges between 1 chance in $1,000,000\left(1 \times 10^{-6}\right)$ to 100 chance in $1,000,000$ $\left(1 \times 10^{-4}\right)$. Non-carcinogenic health effects of $\mathrm{HCH}$ and DDT was estimated as HQ. HQ is the exact measure of the magnitude of exposure potential or a quantifiable potential for developing non-carcinogenic health effects after averaged exposure period. If the $\mathrm{HQ}$ for a chemical is equal to or less than one (1), it is believed that there is no appreciable risk that non-cancer health effects will occur. The potential for noncancer effects was evaluated by comparing the estimated average daily dose $\left(\mathrm{mg} \mathrm{kg}^{-1} \mathrm{~d}^{-1}\right)$ of the pesticide compounds with the RfD ( $\mathrm{mg} \mathrm{kg}^{-1}$ $\mathrm{d}^{-1}$ ) for that compound. Total non-cancer risk was derived simply by summing the $\mathrm{HQ}$ values for individual vegetables. This total $\mathrm{HQ}$ is referred to as HI. If the HI value is less than 1 (one), non-cancer risks are not expected from any chemical, alone or in combination with others. The quantified health HQ for total pesticides (HCH, DDT isomers) through vegetables for human adults has been given in Table 4 . The average $\mathrm{HI}$ was $1.5 \times 10^{-3}, 7.3 \times 10^{-3}, 2.1 \times 10^{-3}$, $5.2 \times 10^{-3}, 1.7 \times 10^{-3}$ and $4.0 \times 10^{-3}$, for carrot, cauliflower, eggplant, radish, spinach and tomato, respectively. These estimated $\mathrm{HI}$ values were much lower than the acceptable safe risk level $(\mathrm{HI} \leq 1)$, indicating negligible risk.

\section{Conclusions}

Concentrations of analysed pesticide compounds in studied vegetables were found within the recommended MRLs. The estimated average daily intakes of HCH and DDT compounds through vegetables were lower than RfD. Subsequently, health risk to humans in terms of HQs and HI was much less than one, suggesting negligible risk. There were numbers of limitations involved in method of risk estimation which may lead to uncertainties in total risk, such as consideration of different age groups. Because, infants and childhood age group may have a greater probability of producing risk than exposure in adulthood. Body weight differences and possible synergistic effects of other toxic chemicals could also lead to uncertainties. Therefore, more indepth studies are recommended, which may also including other persistent organic pollutants and toxic metals.

\section{References}

1. ATSDR (Agency for Toxic Substances and Disease Registry). Toxicological profile for hexachlorocyclohexanes. Atlanta, GA: U S Department of Health \& Human Services Public Health Service, ATSDR; 2005. Available from: http://www.atsdr.cdc.gov/ toxprofiles/tp.asp?id=754\&tid=138

2. ATSDR (Agency for Toxic Substances and Disease Registry). Toxicological profile for DDT, DDE, and DDD. Atlanta, GA: U S Department of Health \& Human Services Public Health Service, ATSDR; 2008.

3. Wania F, Mackay D. Tracking of distribution of persistent organic pollutants. Environ Sci Technol 1996;30:390A-6A.

4. UNEP (United Nations Environment Programme). UNEP/POPS/COP.4/38, 8 May 2009; Available from: http://www.POPs.int/

5. van den Berg H. Global status of DDT and its alternatives for use in vector control to prevent disease. Environ Health Persp 2009;117:1656-63.

6. Jit S, Dadhwal M, Kumari H, Jindal S, Kaur $\mathrm{J}$, et al. Evaluation of hexachlorocyclohexane contamination from the last lindane production plant operating in India. Environ Sci Pollut Res 2011;18:586-97.

7. Government of India. Annual Report 20112012, Department of Chemicals \& Petrochemicals Ministry of Chemicals \& Fertilizers. New Delhi: Government of India; 2012.

8. Bhattacharyya A, Barik SR, Ganguly P. New pesticide molecules, formulation technology and uses: present status and future challenges. J Plant Prot Sci 2009;1:9-15. 
9. UNEP (United Nations Environment Programme). National implementation plans Stockholm Convention on persistent organic pollutants (POPs) (Government of India). Stockholm: Secretariat of the Stockholm Convention; 2011. Available from: http://chm.pops.int/

10. Snedeker SM. Pesticides and breast cancer risk: a review of DDT, DDE, and dieldrin. Environ Health Perspect 2001;109:3547.

11. Ejaz S, Akram W, Lim CW, Lee JJ, Hussain I. Endocrine disrupting pesticides: a leading cause of cancer among rural people in Pakistan. Exper Oncol 2004;26:98-105.

12. Cox S, Niskar AS, Narayan KMV, Marcus M. Prevalence of self-reported diabetes and exposure to organochlorine pesticides among Mexican Americans: hispanic health and nutrition examination survey, 1982-1984. Environ Health Perspect 2007; 115:1747-52.

13. Lee DH, Steffes M, Jacob DR Jr. Positive associations of serum concentration of polychlorinated biphenyls or organochlorine pesticides with self-reported arthritis, especially rheumatoid type, in women. Environ Health Perspect 2007;115:883-8.

14. Mnif W, Hassine AH, Bouaziz A, et al. Effect of endocrine disruptor pesticides: a review. Int $\mathrm{J}$ Environ Res Public Health 2011;8:2265-303.

15. Robison AK, Sirbasku DA, Stancel GM. DDT supports the growth of an estrogenresponsive tumor. Toxicol Lett 1985;27: 109-13.

16. Nakata H, Kawazoe M, Arizono K, Abe S, Kitano T, Shimada H, et al. Organochlorine pesticides and polychlorinated biphenyl residues in foodstuffs and human tissue from China: status of contamination, historical trend, and human dietary exposure. Arch Environ Contam Toxocol 2002;43:473 80.

17. Hamilton D, Ambrus A, Dieterle R, Felsot A, Harris C, Petersen B, et al. Pesticide residues in food-acute dietary exposure. Pest Manage Sci 2004;60:311-39.

18. Wendie L, Claeysa WL, Schmit JF, Bragard C, Maghuin-Rogister G, Pussemier L, et al. Exposure of several Belgian consumer groups to pesticide residues through fresh fruit and vegetable consumption. Food Control 2011;22:508-16.

19. Samanta S. Organochlorine pesticide residue studies in fish of the River Ganges in West Bengal. Pesticide Res J 2006; 18:104-8.

20. Aktar MW, Paramasivam M, Sengupta D, Purkait S, Ganguly M, Banerjee S. Impact assessment of pesticide residues in fish of Ganga River around Kolkata in West Bengal. Environ Monit Assess 2009;157: 97-104.
21. Devanathan G, Subramanian A, Someya M, Sudaryanto A, Isobe T, Takahashi S, et al. Persistent organochlorines in human breast milk from major metropolitan cities in India. Environ Poll 2008;157:148-54.

22. Someya M, Ohtake M, Kunisue T, Subramanian A, Takahashi S, Chakraborty $P$, et al. Persistent organic pollutants in breast milk of mothers residing around an open dumpsite in Kolkata, India: specific dioxin-like PCB levels and fish as a potential source. Environ Int 2009;36:27-35.

23. Guzzella L, Roscioli C, Vigano, L, Saha M, Sarkar SK, Bhattacharya A. Evaluation of the concentration of $\mathrm{HCH}$, DDT, HCB, PCB and $\mathrm{PAH}$ in the sediments along the lower stretch of Hugli estuary, West Bengal, northeast India. Environ Int 2005;31:523-34.

24. Sarkar SK, Binneli A, Riva C, Parolini M, Chatterjee $\mathrm{M}$, Bhattacharya $\mathrm{AK}$, et al. Organochlorine pesticide residues in sediment cores of Sundarban wetland, northeastern part of Bay of Bengal, India, and their ecotoxicological significance. Arch Environ Contam Toxicol 2008;55:358-71.

25. Sarkar SK, Bhattacharya BD, Bhattacharya A, Chatterjee M, Alam A, Satpathy KK, et al. Occurrence, distribution and possible sources of organochlorine pesticide residues in tropical coastal environment of India: an overview. Environ Int 2008; 34:1062-71.

26. Zhang G, Chakraborty P, Li J, Sampathkumar P, Balasubramanian T, Kathiresan K, et al. Passive atmospheric sampling of organochlorine pesticides, polychlorinated biphenyls, and polybrominated diphenyl ethers in urban, rural, and wetland sites along the coastal length on India. Environ Sci Technol 2008;42:8218-23.

27. Chakraborty P, Zhang G, Li J, Xu Y, Liu X, Tanabe $S$, et al. Selected organochlorine pesticides in the atmosphere of major Indian cities: levels, regional versus local variations, and sources. Environ Sci Technol 2010;44:8038-43.

28. Devi NL, Qi S, Chakraborty P, Zhang G, Yadav IC. Passive air sampling of organochlorine pesticides in a northeastern state of India, Manipur. J Environ Sci 2011;23:808-15.

29. Singh B, Gupta A. Monitoring of pesticide residues in farmgate and market samples of vegetables in a semiarid, irrigated area. Bull Environ Contam Toxicol 2002;68:74751.

30. Kumari B, Madan VK, Kumar R, Kathpal TS. Monitoring of seasonal vegetables for pesticide residues. Environ Monit Assess 2002;74:263-70.

31. Kumari B, Kumar R, Madan VK, Singh R, Singh J, Kathpal TS. Magnitude of pesticide contamination in winter vegetables from Hissar, Haryana. Environ Monit
Assess 2003;87:311-8.

32. Mukherjee I. Pesticide residues in vegetables in and around Delhi. Environ Monit Assess 2003;86:265-71.

33. Bhanti M, Taneja A. Monitoring of organochlorine pesticide residues in summer and winter vegetables from Agra, India - a case study. Environ Monit Asses 2005;10:341-6.

34. Ranga Rao GV, Sahrawat KL, Ch Rao S, Das B, Reddy KK, Bharath BS, et al. Insecticide residues in vegetable crops growth in Kothapalli watershed, Andhra Pradesh, India: a case study. Indian J Dryland Agric Res Dev 2009;24:21-7.

35. Srivastava AK, Trivedi P, Srivastava MK, Lohani M, Srivastava LP. Monitoring of pesticide residues in market basket samples of vegetable from Lucknow City, India: QuEChERS method. Environ Monit Assess 2011;176:465-72.

36. Bankar R, Ray AK, Kumar A, Adeppa K, Puri S. Organochlorine pesticide residues in vegetables of three major markets in Uttar Pradesh, India. Acta Biologica Indica 2012;1:77-80.

37. Kumar B, Mukherjee DP. Organochlorine residues in vegetables. Int $\mathrm{J}$ Veg Sci 2012;18:121-36.

38. Gowda SRA, Somashekar RK. Evaluation of pesticide residues in farmgate samples of vegetables in Karnataka, India. Bull Environ Contam Toxicol 2012;89:626-32.

39. Kumari B, Kathpal TS. Monitoring of pesticide residues in vegetarian diet. Environ Monit Assess 2009;151:19-26.

40. Brock JWL, Melnyk J, Caudill SP, Needham LL, Bond AE. Serum levels of several organochlorine pesticides in farmers correspond with dietary exposure and local use history. Toxicol Ind Health 1998;14:275-89.

41. USEPA. Risk assessment guidance for superfund. Human health evaluation manual (Part A). EPA 540-1-89-002. Washington, DC: United States Environmental Protection Agency; 1989.

42. Morra P, Bagli S, Spadoni G. The analysis of human health risk with a detailed procedure operating in a GIS environment. Environ Int 2006;32:444-54.

43. USEPA. Human health risk assessment; 2012. Available from: http://www.epa.gov/ reg3hwmd/risk/human

44. Yang Y, Li D, Mu D. Levels, seasonal variations and sources of OCPs in ambient air of Guangzhou, China. Atm Environ 2008;42:677-87.

45. Willet L, Ulrich EM, Hites HA. Differential toxicity and environmental fates of hexachlorocyclohexane isomers. Environ Sci Technol 1998;32:2197-207.

46. Malaiyandi M, Shah S. Evidence of photoisomerization of hexachlorocyclohexane 
isomers in the ecosphere. J Environ Sci Health 1980;A19:887-910.

47. Walker K, Vallero DA, Lewis RG. Factors influencing the distribution of lindane and other hexachlorocyclohexanes in the environment. Environ Sci Technol 1999;33:4373-8.

48. Hoai PM, Ngoc NT, Minh NH, Viet PH, Berg M, Alder AC, et al. Recent levels of organochlorine pesticides and polychlorinated biphenyls in sediments of the sewer system in Hanoi, Vietnam. Environ Poll 2010;158:913-20.

49. Li YF, Macmillan A, Scholtz MT. Global HCH usage with $1^{0} \times 1^{0}$ longitude/latitude resolution. Environ Sci Technol 1996;30:3525-33.

50. Spencer W, Cliath MM. Volatility of DDT and related compounds. J Agric Food Chem 1972;20:645-9.

51. WHO (World Health Organization). DDT and ifs derivatives- environmental aspects (Environmental Health Criteria 83).
Geneva: World Health Organization; 1989.

52. Travis CC, Arms AD. Bioconcentration of organics in beef, milk, and vegetation. Environ Sci Technol 1988;22:271-4.

53. Atlas E, Giam CS. Ambient concentration and precipitation scavenging of atmospheric organic pollutants. Water Air Soil Pollut 1988;38:19-36.

54. Talekar NS, Sun LT, Lee EM, Chen JS. Persistence of some insecticides in subtropical soils. J Agric Food Chem 1977;25: 348-52.

55. Battu RS, Singh B, Kang BK. Contamination of liquid milk and butter with pesticide residues in the Ludhiana district of Punjab state, India. Ecotoxicol Environ Safe 2004;9:324-31.

56. Sharma VP. Malaria and poverty in India. Curr Sci 2003;84:513-5.

57. European Commission. Commission Regulation (EC) No 149/2008 of 29 January 2008 amending Regulation (EC)
No 396/2005 of the European Parliament and of the Council by establishing Annexes II, III and IV setting maximum residue levels for products covered by Annex I thereto (Text with EEA relevance). In: Official Journal L 58, 1/3/2008, pp 1398. Available from: http://eur-lex.europa. eu/LexUriServ/LexUriServ.do?uri=CELEX: 32008R0149:EN:NOT

58. FSSAI (Food Safety Standards Authority of India). Food safety and standards regulations; 2011. Available from: http://www. fssai.gov.in

59. WHO. Inventory of IPCS and other WHO pesticide evaluations and summary of toxicological evaluations performed by the Joint Meeting on Pesticide Residues (JMPR) through 2009. Geneva: World Health Organization; 2009. Available from: http://www.who.int 